\title{
Two nucleon correlations measured with ${ }^{3} \mathrm{He}(\mathrm{e}, \mathrm{e} \mathrm{pp}) \mathrm{n}$
}

\author{
L.B. Weinstein and R. Niyazov ${ }^{\mathrm{a}}$, for the CLAS Collaboration \\ Old Dominion University, Norfolk, VA 23529, USA
}

Received: 18 Jul 2003 / Accepted: 14 Nov 2003 /

Published Online: 6 Feb 2004 - (C) Società Italiana di Fisica / Springer-Verlag 2004

\begin{abstract}
Despite tremendous progress in recent years in measuring single nucleon momentum distributions in nuclei, two-nucleon momentum distributions remain largely unmeasured. Here we report on a measurement of ${ }^{3} \mathrm{He}(\mathrm{e}, \mathrm{e}$ 'pp $) \mathrm{n}$ that is primarily sensitive to the two-nucleon momentum distribution and relatively uncontaminated by final state rescattering or by two-body currents.
\end{abstract}

PACS. 21.45. $+\mathrm{v}-25.30 . \mathrm{Dh}$

\section{Introduction}

Single nucleon properties in nuclei have been thoroughly studied, primarily through proton knockout from nuclei, such as $\mathrm{O}\left(e, e^{\prime} p\right)$ [1]. The valence proton momentum distributions and more deeply bound proton momentum and energy distributions have been measured by many experiments [2]. Valence knockout is well described by single nucleon knockout calculations that include the effects of final state interactions. However, the fact that a) we only see about $70 \%$ of the 'expected' number of nucleons and b) there is a large cross section at large excitation energies of the residual nucleus indicates that multi-nucleon processes play a significant role. While there have been many $\left(e, e^{\prime} p\right)$ measurements from nuclei, there have been very few $\left(e, e^{\prime} p p\right)$ measurements.

There are two general sources of two-nucleon knockout from nuclei. One source is the interaction of the virtual photon with two nucleons, typically through meson exchange currents or isobar configurations. This is referred to as a two-body current. The second one is the interaction of the virtual photon with one nucleon of a 'correlated pair'. (Note that the distinction between correlations [in the wave function] and currents [in the operator] is blurred by the fact that unitary transformations can transform one into the other.)

A correlated pair is typically a pair of nucleons that have large relative momentum (because they are at short range) and small total momentum. Thus, one signature of correlations is finding two nucleons with large relative momentum and small total momentum in the initial state. Unfortunately, the effects of $N N$ correlations are frequently obscured by the effects of two body currents [3]. In order to disentangle these competing effects, a series of comprehensive measurements are needed.

\footnotetext{
a Present address: Jefferson Lab, Newport News, VA 23606
}
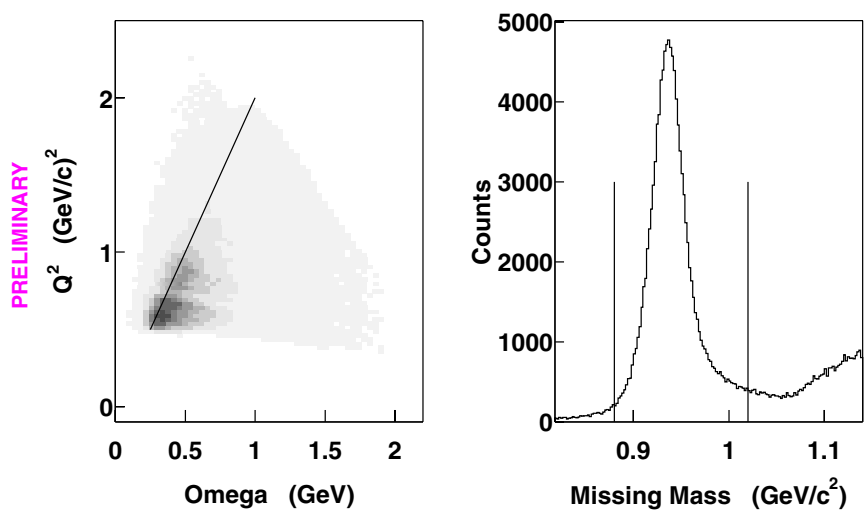

Fig. 1. a $Q^{2}$ vs $\omega$ for ${ }^{3} \mathrm{He}\left(\mathrm{e}, \mathrm{e}^{\prime} \mathrm{pp}\right) \mathrm{n}$ at $E_{\text {beam }}=2.2 \mathrm{GeV}$. Note the huge kinematic acceptance. $\mathbf{b}$ Missing mass for ${ }^{3} \mathrm{He}\left(\mathrm{e}, \mathrm{e}^{\prime} \mathrm{pp}\right)$. We cut at the indicated lines to select (e,e'pp)n events

The seven experiments took data simultaneously in Spring 1999, measuring approximately 500 million $\mathrm{A}\left(e, e^{\prime} X\right)$ events, using the CEBAF Large Acceptance Spectrometer (CLAS) at Jefferson Lab, a $4 \pi$ magnetic spectrometer, with 1.16, 2.26 and $4.46 \mathrm{GeV}$ polarized electrons incident on targets from ${ }^{3} \mathrm{He}$ to ${ }^{56} \mathrm{Fe}$ [4]. This paper reports the results from 2.2 and $4.4 \mathrm{GeV}$ electrons on ${ }^{3} \mathrm{He}$.

\section{Measuring ${ }^{3} \mathrm{He}\left(e, e^{\prime} p p\right) n$}

We studied ${ }^{3} \mathrm{He}\left(\mathrm{e}, \mathrm{e}^{\prime} \mathrm{pp}\right) \mathrm{n}$ by measuring electron induced two proton knockout reactions from ${ }^{3} \mathrm{He}$ using the CLAS detector and cutting on the missing mass. Figures $1 \mathrm{k}$ and b show the electron acceptance and undetected neutron missing mass resolution for $E_{\text {beam }}=2.2 \mathrm{GeV}$. The threshold of the CLAS is approximately $250 \mathrm{MeV} / \mathrm{c}$ for protons.

Because this is the first time that ${ }^{3} \mathrm{He}\left(\mathrm{e}, \mathrm{e}^{\prime} \mathrm{pp}\right) \mathrm{n}$ has been measured using an almost $4 \pi$ detector, our data anal- 

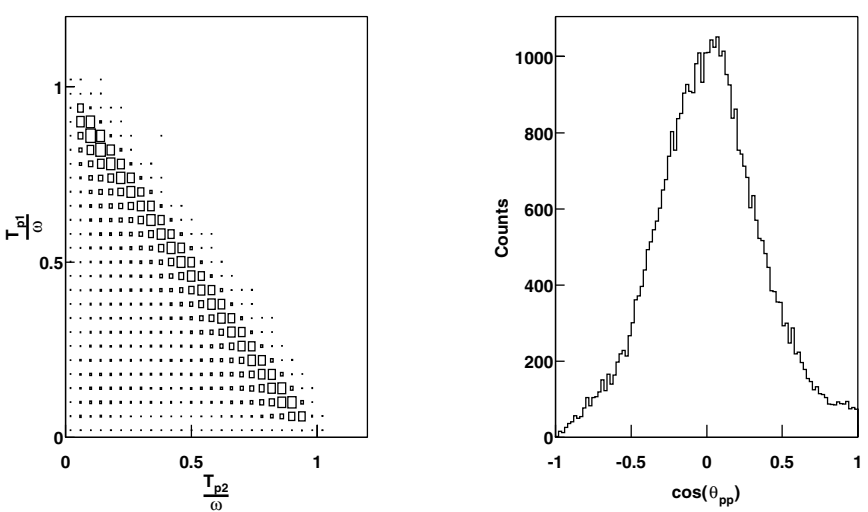

Fig. 2. a Nucleon kinetic energy distribution for $2.2 \mathrm{GeV}$ ${ }^{3} \mathrm{He}\left(\mathrm{e}, \mathrm{e}^{\prime} \mathrm{pp}\right) \mathrm{n}$. The kinetic energy of proton 1 divided by $\omega$ $\left(T_{p 1} / \omega\right)$ is plotted versus the same for proton 2 . (Note $p_{p} \geq 250$ $\mathrm{MeV} / \mathrm{c}$.) Note the dominant band running from $(1,0)$ to $(0,1)$ corresponding to very low energy neutrons. $\mathbf{b}$ The cosine of the opening angle between the two protons for events with $p_{n}<250 \mathrm{MeV} / \mathrm{c}$. Note the large peak at $90^{\circ}$

ysis philosophy is to follow and understand the dominant features of the data.

In order to understand the energy sharing in the reaction, we plotted the kinetic energy divided by the energy transfer of the first proton $\left(T_{p 1} / \omega\right)$ versus that of the second proton $\left(T_{p 2} / \omega\right)$ for each event (a lab-frame Dalitz plot). Note that the assignment of protons 1 and 2 is arbitrary. See Fig. 2 k. The threshold for proton detection in CLAS is $p_{p} \geq 250 \mathrm{MeV} / \mathrm{c}$. The dominant feature of this plot is a band running from the upper left corner (proton 1 has almost all of the energy) to the lower right (proton 2 has almost all of the energy) corresponding to very low energy neutrons. When we cut on this ridge, requiring that $p_{n}<250 \mathrm{MeV} / \mathrm{c}$, the opening angle between the two protons peaks at $90^{\circ}$ (see Fig. 2 $\mathrm{b}$ ). As we know from teaching introductory physics, when a moving object collides elastically with an identical stationary object, the opening angle is always $90^{\circ}$. Thus, this peak indicates single proton knockout followed by hard $p p$ rescattering.

Further evidence for this comes from preliminary data from Zhang [6]. He analyzed the same ${ }^{3} \mathrm{He}(\mathrm{e}, \mathrm{e}$ 'pp)n data with $p_{n}<150 \mathrm{MeV} / \mathrm{c}$, looking at events with a fast backward proton $\left(\theta_{p q}>100^{\circ}\right)$. See Fig. 3, It was conjectured that these fast backward protons could not have been affected by final state interactions (FSI). However, calculations by Laget show that the cross section is dominated by final state interactions with significant contributions from two-body currents (meson exchange currents and isobar configurations). These results are very consistent with the $90^{\circ}$ peak in the $p p$ opening angle distribution.

Since we are looking for two-nucleon correlations, we eliminate these FSI-dominated events by setting the same threshold for neutrons as for protons: $p_{n} \geq 250 \mathrm{MeV} / \mathrm{c}$. When we look at this Dalitz plot (see Fig. 4) we see three peaks at the three corners of the plot, corresponding to events where two 'fast' nucleons each have less than $20 \%$ of the energy transfer and the third 'leading' nucleon has the remainder. We call the two nucleons 'fast' because

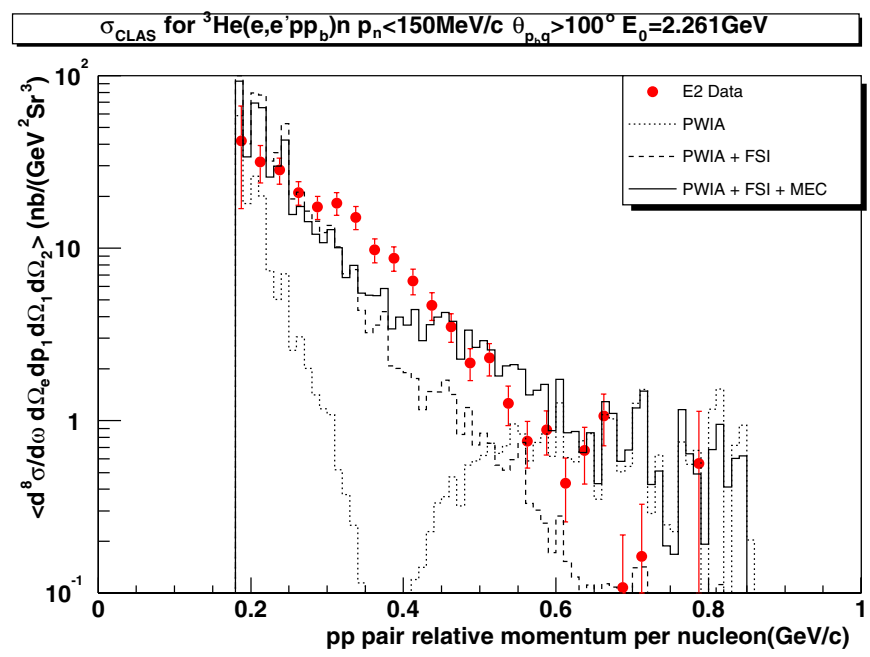

Fig. 3. Preliminary cross section vs $p p$ relative momentum $\mathbf{p}_{\text {rel }}=\left[\left(\mathbf{p}_{\text {forward }}-\mathbf{q}\right)-\mathbf{p}_{\text {backward }}\right] / 2$ for ${ }^{3} \mathrm{He}(\mathrm{e}, \mathrm{e}$ 'pp $) \mathrm{n}$ events with a fast backward proton and a slow neutron. The points show the data, the dotted line is the PWIA calculation, the dashed line also includes FSI and the solid line also includes two-body currents. All calculations are by Laget

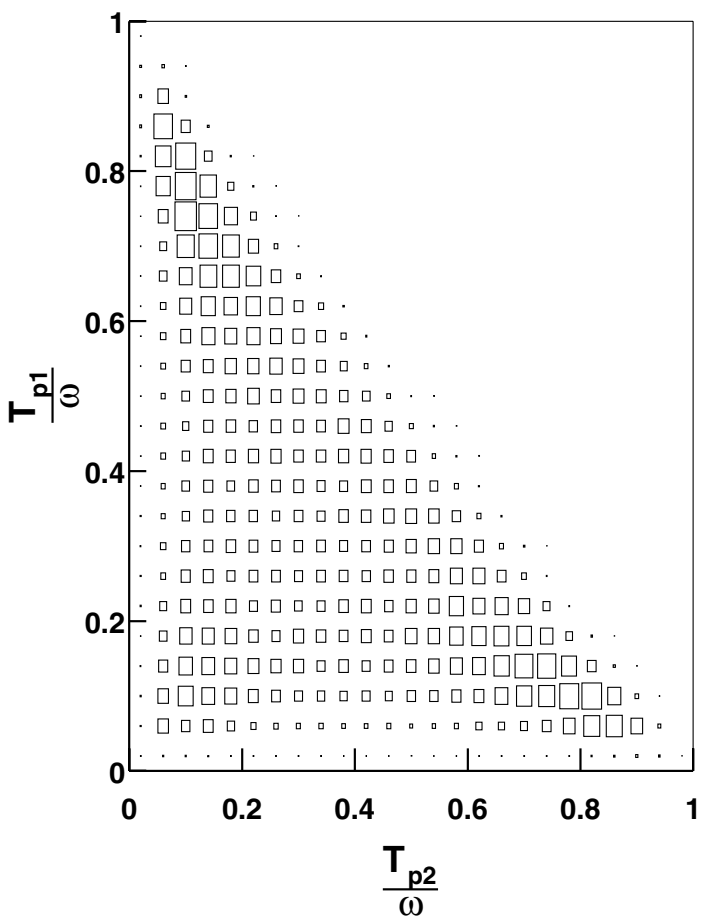

Fig. 4. Nucleon kinetic energy distribution for $2.2 \mathrm{GeV}$ ${ }^{3} \mathrm{He}\left(\mathrm{e}, \mathrm{e}^{\prime} \mathrm{pp}\right) \mathrm{n}$. The kinetic energy of proton 1 divided by $\omega$ $\left(T_{p 1} / \omega\right)$ is plotted versus the same for proton 2 . (Note $p_{p}, p_{n} \geq$ $250 \mathrm{MeV} / \mathrm{c}$.) Note the peaks in the corners

$p \gg p_{\text {fermi }}$. These peaks are much more pronounced at $E_{\text {beam }}=4.4 \mathrm{GeV}$ (not shown). We cut on these peaks where the two fast nucleons each have less than $20 \%$ of the energy transfer.

Then we looked at the opening angle of the two fast nucleons. Figure 5 ta shows the pair opening angle for fast 

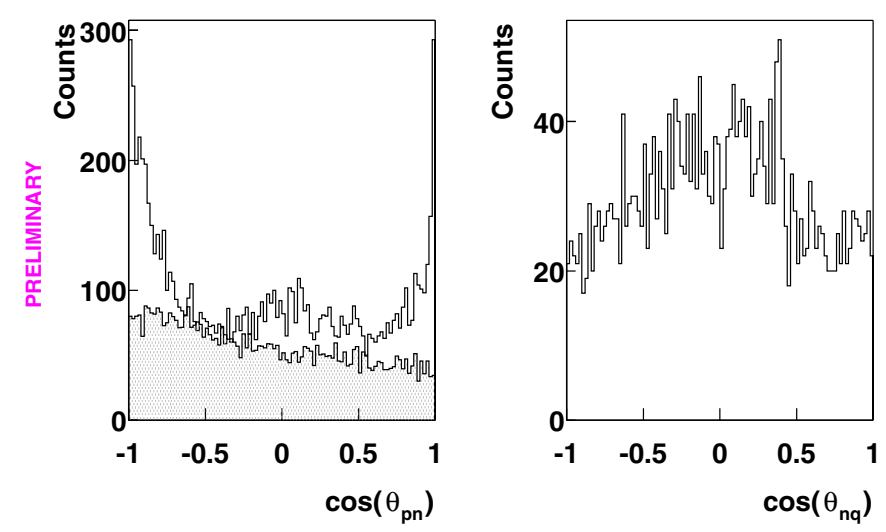

Fig. 5. a Opening angle of the fast $p n$ pairs for events in the upper left and lower right corners of Fig. $4 \mathrm{~b}$. The histogram shows the data, the filled histogram shows the fire ball phase space simulation (with arbitrary normalization). b The angle between the neutron in the fast $p n$ pair and $\mathbf{q}$ where $p_{\perp}<300$ $\mathrm{MeV} / \mathrm{c}$

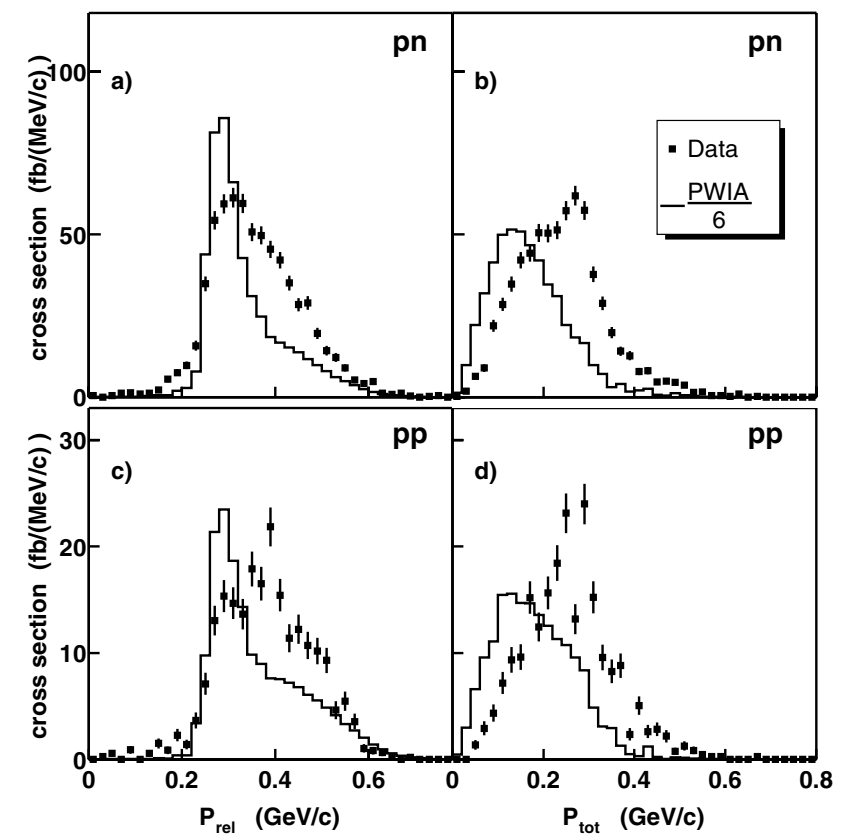

Fig. 6. $2.2 \mathrm{GeV}{ }^{3} \mathrm{He}\left(e, e^{\prime} p p\right) n$ cross section vs. momentum. Points $=$ data, histogram $=$ PWIA decreased by a factor of 5 . a leading proton and a fast $p n$ pair: pair relative momentum $p_{r e l}$; b leading proton and a fast $p n$ pair: total momentum $p_{t o t}$; c leading neutron and a fast $p p$ pair: $p_{r e l}$; d leading neutron and a fast $p p$ pair: $p_{\text {tot }}$

pn pairs with a leading proton. Note the large peak at 180 degrees $\left(\cos \theta_{N N} \approx-1\right)$. The distribution for fast $p p$ pairs with a leading proton is identical. The peak is not due to the cuts, since we do not see it in a fire ball phase space simulation assuming three body absorption of the virtual photon and phase space decay. It is also not due to the CLAS acceptance since we see it both for leading protons (which we detect) and leading neutrons (which we infer from missing mass). This back-to-back peak is a strong indication of correlated $N N$ pairs.

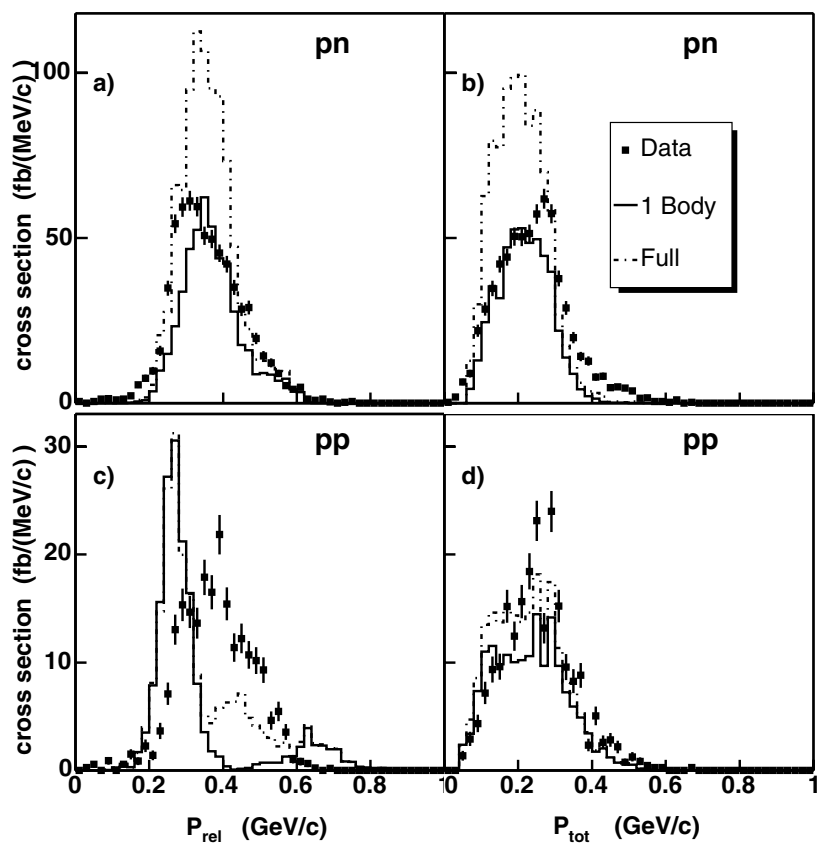

Fig. 7. $2.2 \mathrm{GeV}{ }^{3} \mathrm{He}\left(e, e^{\prime} p p\right) n$ cross section vs. momentum. Points $=$ data, solid histogram $=$ Laget 1-body calculation, dot-dashed histogram $=$ Laget full calculation. a leading proton and a fast $p n$ pair: pair relative momentum $p_{\text {rel }}$; $\mathbf{b}$ leading proton and a fast $p n$ pair: total momentum $p_{t o t}$; c leading neutron and a fast $p p$ pair: $p_{r e l} ; \mathbf{d}$ leading neutron and a fast $p p$ pair: $p_{t o t}$

\section{Studying correlated pairs}

Now consider these presumably correlated pairs. Since we believe that we have observed events where the leading nucleon absorbed the virtual photon and the two fast nucleons are emitted back to back, we cut on the perpendicular momentum of the leading nucleon to deemphasize rescattering $\left(p_{\perp}<300 \mathrm{MeV} / \mathrm{c}\right)$. This cut selects the backto-back events very cleanly. Unfortunately, there are only 3400 fast $p n$ and 1100 fast $p p$ events remaining in the entire $2.2 \mathrm{GeV}$ data set (and ten times fewer at $4.4 \mathrm{GeV}$ ).

If the fast back-to-back $N N$ pairs are really uninvolved in the photon absorption, then they should be distributed isotropically (the angular distribution of the neutrons with respect to $\mathbf{q}$ is shown in Fig. [5b). Further evidence that the fast $N N$ pair is a spectator comes from the average momentum of the pair along $\mathbf{q}$. This is about $0.07 \mathrm{GeV} / \mathrm{c}$ for $E_{\text {beam }}=2.2 \mathrm{GeV}$ and about $0.1 \mathrm{GeV} / \mathrm{c}$ for $E_{\text {beam }}=$ $4.4 \mathrm{GeV}$, much less than the average momentum transfers of $Q^{2}=0.7$ and $1.4(\mathrm{GeV} / \mathrm{c})^{2}$ respectively.

The $2.2 \mathrm{GeV}$ fast $N N$ pair relative $\left(p_{\text {rel }}=\frac{1}{2}\left|\mathbf{p}_{1}-\mathbf{p}_{2}\right|\right)$ and total $\left(p_{\text {total }}=\left|\mathbf{p}_{1}+\mathbf{p}_{2}\right|\right)$ momentum distributions are shown in Fig. 6. Note how similar the $p p$ and $p n$ distributions are. The $4.4 \mathrm{GeV}$ distributions (not shown) are similar.

Thus, because when we select a quasifree leading nucleon, the fast $N N$ pairs are back to back, relatively isotropic and have small average momentum along $\mathbf{q}$, we conclude that the fast $N N$ pair is a spectator. Because 
we measure similar $p_{\text {total }}$ and $p_{\text {rel }}$ distributions for $p p$ and $p n$ pairs and $0.5<Q^{2}<1\left(E_{\text {beam }}=2.2 \mathrm{GeV}\right)$ and $1<Q^{2}<2(\mathrm{GeV} / \mathrm{c})^{2}\left(E_{\text {beam }}=4.4 \mathrm{GeV}\right)$, we conclude that we have measured bound state $N N$ correlations.

We appear to have measured $N N$ correlations in ${ }^{3} \mathrm{He}$ by striking the third nucleon and detecting the correlated pair. This is similar to other proposed correlation searches where you strike one nucleon of a correlated pair and detect the other nucleon leaving the nucleus. However, these other searches suffer from the weakness that their proposed signal can also be due to two body currents (eg: photon absorption on an exchanged meson) (see Fig. 3).

\section{Comparison to theory}

Calculations by W. Glöckle [7 at lower energy strengthen this conclusion. He calculated the ${ }^{3} \mathrm{He}\left(\mathrm{e}, \mathrm{e}^{\prime} \mathrm{pp}\right) \mathrm{n}$ cross section where the leading nucleon has momentum $\mathbf{p}_{N}=\mathbf{q}$ and the other two nucleons have total momentum $p_{\text {total }}=$ 0 for various values of the momentum transfer, $400 \leq|\mathbf{q}| \leq$ $600 \mathrm{MeV} / \mathrm{c}$, and relative momentum. He found that MEC did not contribute, rescattering of the leading nucleon did not contribute, and the continuum state interaction of the outgoing $N N$ pair decreased the cross section by a factor of approximately 10 relative to the PWIA result. Thus, he found that this reaction is a very clean way to measure the overlap integral between the $N N$ continuum state and the same two nucleons in the bound state.

C. Ciofi degli Atti and L. Kaptari also found that the continuum interaction of the outgoing pair significantly decreased the cross Sect. 8 .

We compared our results to two other calculations, 1) a Plane Wave Impulse Approximation (PWIA) calculation by M. Sargsian 9 using Glöckle's bound state wave function with no final state interactions, and 2) a diagrammatic calculation by J.-M. Laget [10,11/12 using a Faddeev wave function from P. Sauer and including one-, two-, and three- body mechanisms as well as rescattering terms. We averaged all of the models over the CLAS acceptances and cuts using a monte carlo.

The PWIA calculation of Sargsian has $Q^{2}$ vs $\omega, N N$ pair opening angle, $p_{\text {rel }}$, and $p_{\text {total }}$ distributions that are consistent with the data (see Fig. 6). The momentum distributions peak at smaller momentum than the data. This is probably due to a much stronger $N N$-pair continuum interaction in the $N N$ s-wave than in the p-wave. This interaction therefore reduces the s-wave strength more than the p-wave, shifting the peaks to higher momenta. The PWIA cross section is a factor of 5 larger than the data which is consistent with the expected effects of the $N N$ continuum state interaction calculated by Glöckle, by Ciofi degli Atti and by Laget. It also predicts the same ratio of $p p$ to $p n$ pairs as seen in the data.

Laget's one-body calculations describe the $p n$ pairs well, both qualitatively and quantitatively (see Fig. 7, and $\mathrm{b}$ ). However, the full calculation overestimates the data by about $60 \%$. The calculation describes $p_{\text {rel }}$ for $p p$ pairs badly but $p_{t o t}$ well (see Fig. 7 t and d). The failure is due possibly to the truncation of the wave function to only the lower angular momentum states. Note that Laget predicts three-body effects to be much larger for events with a leading proton and a fast $p n$ pair than for events with a leading neutron and a fast $p p$ pair. We do not see this difference in the data.

\section{Summary}

We have studied the ${ }^{3} \mathrm{He}\left(\mathrm{e}, \mathrm{e}^{\prime} \mathrm{pp}\right) \mathrm{n}$ reaction, selecting events where one nucleon has most of the kinetic energy and has less than $300 \mathrm{MeV} / \mathrm{c}$ of momentum perpendicular to q. When we do this, we see isotropic, back-to-back, fast $N N$ pairs with small average momentum along $\mathbf{q}$. We have measured the total and relative momentum distributions of these pairs and found that they do not depend significantly on isospin ( $p p$ vs $p n$ pairs) or on momentum transfer.

PWIA calculations reproduce the observed $p p$ to $p n$ cross section ratio, indicating the importance of singlenucleon knockout mechanisms. Calculations by Laget with many different diagrams and a truncated bound state wave function predict that leading-nucleon FSI and twobody exchange currents are negligible, and continuumstate interactions of the spectator pair reduce the cross section significantly. However, the predicted three-body exchange current contributions of about $20 \%$ for $p p$ pairs and $50 \%$ for $p n$ pairs do not improve agreement with the data.

Thus, by measuring ${ }^{3} \mathrm{He}\left(\mathrm{e}, \mathrm{e}^{\prime} \mathrm{pp}\right) \mathrm{n}$, we appear to have directly measured $N N$ correlations without any significant contamination from other processes by striking the third nucleon and detecting the spectator correlated pair.

Acknowledgements. I thank Claudio Ciofi degli Atti, Walter Glockle, Jean-Marc Laget and Misak Sargsian for their calculations and physics insight. This work was supported by a grant from the US Department of Energy.

\section{References}

1. J. Gao et al.: Phys. Rev. Lett. 84, 3265 (2000)

2. J. Kelly: Adv. Nucl. Phys. 23, 75 (1996)

3. S. Janssen et al.: Nucl. Phys. A 672, 285 (2000)

4. B. Mecking et al.: Nucl. Inst. and Meth. A 503, (2003)

5. M. Mestayer et al.: Nucl. Inst. and Meth. A 449, 81 (2000)

6. B. Zhang: Ph.D. thesis, MIT (2002)

7. W. Glöckle et al.: Acta Phys. Polon. B 32, 3053 (2001), nucl-th/0109070

8. C. Ciofi degli Atti and L. Kaptari: Phys. Rev. C 66, 044004 (2002), nucl-th/0203041

9. M. Sargsian: Private communication

10. J. Laget: J. Phys. G 14 (1988)

11. G. Audit et al.: Nucl. Phys. A 614, 461 (1997)

12. J. Laget: Phys. Rev. C 35 (1987) 